\title{
Syntheses of phenylbutanoid and dienone derivatives and their anti-inflammatory activity
}

\author{
Preeyarat Onkum ${ }^{\mathrm{a}}$, Thitinun Karpkird ${ }^{\mathrm{a}}$, Mesayamas Kongsema ${ }^{\mathrm{b}}$, Weerasak Taengphan ${ }^{\mathrm{c}}$, \\ Theerachart Leepasert ${ }^{\mathrm{a}, *}$ \\ ${ }^{a}$ Department of Chemistry, Faculty of Science, Kasetsart University, Bangkok 10900 Thailand \\ b Department of Zoology, Faculty of Science, Kasetsart University, Bangkok 10900 Thailand \\ c Expert Centre of Innovative Herbal Products, Thailand Institute of Scientific and Technological \\ Research, Pathum Thani 12120 Thailand
}

*Corresponding author, e-mail: fscitcl@ku.ac.th

\begin{abstract}
A series of phenylbutanoid 4a-e and dienone 5a-e derivatives were synthesized and characterized by spectroscopic methods. Cytotoxic and anti-inflammatory activities of the synthesized derivatives were investigated at a potentially non-toxic concentration of $15.63 \mu \mathrm{M}$ (as indicated by cell viability of more than $70 \%$ ) by measuring the nitric oxide content produced by lipopolysaccharide-stimulated RAW264.7 macrophage cells. Compounds 4b, 4e, 5a, $5 \mathrm{~b}$, and $5 \mathrm{e}$ at $15.63 \mu \mathrm{M}$ showed a higher anti-inflammatory activity than the diclofenac drug without affecting cell viability of RAW264.7 macrophage cells. In particular, 5a and 5e showed a nitric oxide inhibition of more than $80 \%$.
\end{abstract}

KEYWORDS: nitric oxide inhibition, anti-inflammation, phenylbutanoid, dienone

\section{INTRODUCTION}

Nonsteroidal anti-inflammatory drugs (NSAIDs) are used for treatment of chronic inflammation and to relieve mild to moderate inflammatory symptoms. Examples of NSAIDs are: naproxen, ibuprofen, aspirin, and diclofenac. However, NSAIDs are associated with a risk of many adverse effects [1], the most important of which are gastrointestinal, cardiovascular, and liver adverse effects. In addition, NSAIDs are associated with an increased risk for chronic kidney disease (CKD). A long-term use of diclofenac and aceclofenac was also reported to increase the risk of stroke by $64 \%$ after two years $[2,3]$. Therefore, NSAIDs should be prescribed and used with caution to avoid adverse effects. Alternative treatments using herbs and herbal products, such as ginger, turmeric, cannabis, cassumunar ginger, etc., are available to help relieve symptoms.

Zingiber cassumunar Roxb., also known as Plai, is a medicinal plant normally found in Thailand. Its chemical constituents and biological activities have been studied, and the essential oil extracted from the plant's rhizome has proven to be useful for health. The main components identified in Plai are $\alpha$-terpinene, $\gamma$-terpinene, terpinen-4-ol, sabinene, and (E)-4(3,4-dimethoxyphenyl)butadiene (DMPBD) $[4,5]$.
It was reported that phenylbutanoids in the rhizome can reduce inflammation, and DMPBD showed inhibition in TPA (12-O-tetradecanoylphorbol13-acetate)-induced edema 11 times higher than diclofenac [6]. Furthermore, the enriched phenylbutanoids from $Z$. cassumunar extracts showed stronger anti-inflammatory activity than each individual phenylbutanoids.

The dienone derivatives are analogs of curcumin. The curcumin was first isolated from turmeric and exhibited various biological activities. Thus, several curcumin analogs were synthesized. The biological activities of curcuminoids and synthetic curcumin analogs were studied, and the results indicateed anticancer and antioxidant activities with low toxicity to cells [7-9].

RAW264.7 macrophage cells play a role in the immune system as the first defence mechanism against invasion of unusual materials and inflammation $[10,11]$. Macrophages could be activated in the inflammatory process by lipopolysaccharide (LPS). Activated macrophages release several different chemical mediators including nitric oxide (NO), an important inflammatory mediator $[12,13]$. Thus, NO production is important in the study of antiinflammation activity, via the Griess reagent assay using nitrite content accumulated in the culture medium as an indicator of NO production [14]. 
In this study, we reported the synthesis of a series of phenylbutanoids and dienone derivatives and the results of cytotoxicity and anti-inflammatory activities of these compounds from our investigation.

\section{MATERIALS AND METHODS}

\section{Chemicals and reagents}

The chemicals for all syntheses were of analytical grade and purchased from Sigma-Aldrich (Singapore) and commercial suppliers, S.M. Chemical and Chemical Express (Bangkok Thailand). The solvents were analytical or HPLC grade and purchased from RCI Labscan (Bangkok, Thailand). The RAW264.7 mouse monocyte macrophage cell line was purchased from the American Type Culture Collection (ATCC) (VA, USA). Lipopolysaccharide (LPS) from Escherichia coli was purchased from Sigma-Aldrich (MO, USA). Dulbecco's Modified Eagle Medium (DMEM, GIBCO ${ }^{8}$ ), foetal bovine serum (FBS, GIBCO $\AA$ ), and penicillin-streptomycin (GIBCO $($ ) were purchased from Biowest (MO, USA). The cell proliferation colorimetric assay was purchased from Biovision (CA, USA). Thin layer chromatography (TLC) aluminium sheets silica gel $60(0.2 \mathrm{~mm}$ thick) and silica gel 60 (70-230 mesh ASTM) for column chromatography were purchased from Merck (Darmstadt, Germany).

${ }^{1} \mathrm{H}$ and ${ }^{13} \mathrm{C}$-NMR spectra were recorded on a Brucker $400 \mathrm{MHz}$ AVANCE III HD spectrometer operating at $400 \mathrm{MHz}$ for ${ }^{1} \mathrm{H}-\mathrm{NMR}$ and $100 \mathrm{MHz}$ for ${ }^{13} \mathrm{C}$-NMR. The chemical shifts $(\delta)$ were reported in $\mathrm{ppm}$ relative to tetramethylsilane (TMS) at the Department of Chemistry, IR spectra were recorded on a Bruker Vertex $70\left(4000-400 \mathrm{~cm}^{-1}\right)$ at the Department of Materials Science, high resolution mass spectra (HRMS) were recorded on a Bruker microTOF-Q III mass spectrometer at the Scientific Equipment Centre, Faculty of Science, Kasetsart University.

\section{General procedure for synthesis of phenylbutan-} oid derivatives $[15,16]$

The synthesis of (E)-4-(substituted phenyl)but-3en-2-one (2a-e) [17]

Firstly, $10 \% \mathrm{NaOH}$ solution $(20 \mathrm{ml})$ was added to a solution of aldehyde (1a-e) $(12 \mathrm{mmol})$ in acetone $(15 \mathrm{ml})$, and the reaction mixture was stirred at room temperature for $0.5-1 \mathrm{~h}$, depending on the starting compounds. Completion was confirmed by TLC. The reaction mixture was adjusted to $\mathrm{pH} 7$ with $3 \mathrm{M} \mathrm{HCl}$, then extracted with dichloromethane, dried over $\mathrm{Na}_{2} \mathrm{SO}_{4}$, and the excess dichloromethane was removed under reduced pressure. The residue obtained was recrystallized from ethanol as compounds $2 \mathrm{a}-\mathrm{e}$.

The synthesis of (E)-4-(substituted phenyl)but-3en-2-ol (3a-e)

Sodium borohydride $(8 \mathrm{mmol})$ was added to a solution of compound $2 \mathrm{a}-\mathrm{e}(4 \mathrm{mmol})$ in absolute methanol $(30 \mathrm{ml})$, and the reaction mixture was stirred overnight at room temperature. The reaction mixture was then extracted with dichloromethane, dried over $\mathrm{Na}_{2} \mathrm{SO}_{4}$, and the excess dichloromethane was removed under reduced pressure. The residue was purified by column chromatography using ethyl acetate:hexane as eluent to obtain compounds $3 a-e$.

\section{The synthesis of (E)-1-(3,4-substituted phenyl) butadiene ( $4 a-e)$}

Firstly, $50 \%$ sulfuric acid $(0.4 \mathrm{ml})$ was added dropwise to a solution of compound 3a-e $(2 \mathrm{mmol})$ in dioxane $(85 \mathrm{ml})$, and the reaction mixture was stirred at $40{ }^{\circ} \mathrm{C}$ for $25 \mathrm{~min}$. The reaction mixture was then diluted with saturated aqueous $\mathrm{NaHCO}_{3}$ solution $(50 \mathrm{ml})$, allowed to cool at room temperature, extracted with dichloromethane, dried over $\mathrm{Na}_{2} \mathrm{SO}_{4}$, and the excess dichloromethane was removed under reduced pressure. The residue was purified by column chromatography using ethyl acetate:hexane as eluent to obtain compounds $4 a-e$.

The synthesis of (1E,4E)-1,5-bis(substituted phenyl)penta-1,4-dien-3-one (5a-e) [18]

Aldehyde (1a-e) $(7 \mathrm{mmol})$ and acetone $(3.5 \mathrm{mmol})$ were mixed in ethanol $(20 \mathrm{ml})$. The mixture was treated with $10 \% \mathrm{NaOH}$ solution $(5 \mathrm{ml})$ and continuously stirred overnight at room temperature. The mixture was adjusted to $\mathrm{pH} 7$ with $3 \mathrm{M} \mathrm{HCl}$ and filtered. The precipitate was recrystallized by $50 \%$ ethanol to obtain compounds $5 \mathrm{a}-\mathrm{e}$.

\section{Preparation of test samples}

The test samples were diluted using two-fold series dilutions from 15.63 to $500 \mu \mathrm{M}$ with DMEM.

\section{Culturing and maintaining of RAW264.7 cell line}

The RAW264.7 cells were cultured and maintained in DMEM containing $10 \%(\mathrm{v} / \mathrm{v})$ heat-inactivated FBS and $1 \%(\mathrm{v} / \mathrm{v})$ penicillin-streptomycin in a 5\% $\mathrm{CO}_{2}$ humidified atmosphere incubator at $37^{\circ} \mathrm{C}$. Cells were then seeded in 96-well plates at a density of $2 \times 10^{4}$ cells/well and incubated for $24 \mathrm{~h}$ before being used. 


\section{Determination of cytotoxic activity}

RAW264.7 cells were treated with various concentrations of samples for $24 \mathrm{~h}$. The highly cytotoxic compound Mitomycin C (MMC), at $20 \mu \mathrm{g} / \mathrm{ml}$, was used as a positive control. After completion of incubation, $100 \mu \mathrm{l}$ of WST (Water Soluble Tetrazolium Salt) solution was added to each well. The plates were kept in darkness for 30 min before measuring the absorbance at $450 \mathrm{~nm}$ using the microplate reader system.

Values of three independent WST assay experiments [19] were used to calculate the percentage viability of the cells. A graph of absorbance ( $Y$-axis) plotted against sample concentration ( $X$-axis) was constructed. The cytotoxicity of samples was presented as $50 \%$ inhibitory concentration $\left(\mathrm{IC}_{50}\right.$ ).

\section{Determination of anti-inflammatory activity}

RAW264.7 cells were treated with $100 \mu \mathrm{l}$ of the samples and incubated for $24 \mathrm{~h}$. Culture media was replaced with $100 \mu \mathrm{l} \mathrm{LPS}$ at $10 \mu \mathrm{g} / \mathrm{ml}$ and incubated for an additional $24 \mathrm{~h}$. After incubation, nitrite content in the culture medium was analysed as an indicator of NO production.

The culture medium was mixed with $100 \mu \mathrm{l}$ Griess reagent ( $1 \%$ sulphanilamide, $5 \%$ phosphoric acid, and $0.1 \%$ naphthyletylene diamine dihydrochloride) and then incubated at room temperature and protected from light for $10 \mathrm{~min}$. After that, the absorbance at $540 \mathrm{~nm}$ was measured using a microplate reader. The amount of nitrite presented in the culture medium was calculated from the sodium nitrite serial dilution standard curve.

\section{Statistical analysis}

All data were expressed as mean \pm standard error (Mean \pm SE) of triplicate determinations.

\section{RESULTS AND DISCUSSION}

The phenylbutanoid derivatives 4a-e were achieved with a general three step reaction. The ketone intermediates $2 \mathrm{a}-\mathrm{e}$ were obtained in $79-95 \%$ yields by the Aldol condensation between substituted commercially available benzaldehydes 1a-e and acetone in basic condition, follow by the reduction of ketone intermediates $2 \mathrm{a}$-e with $\mathrm{NaBH}_{4}$ to give alcohols 3a-e in $81-92 \%$ yields. In the final step, compounds 3a-e underwent dehydration reaction with $50 \% \mathrm{H}_{2} \mathrm{SO}_{4}$, and the reaction was refluxed at $40^{\circ} \mathrm{C}$ to produce desired dienes 4 a-e in 10 $45 \%$ yields. The dienone derivatives $5 \mathrm{a}-\mathrm{e}$ were prepared by Aldol condensation between aldehydes (a)

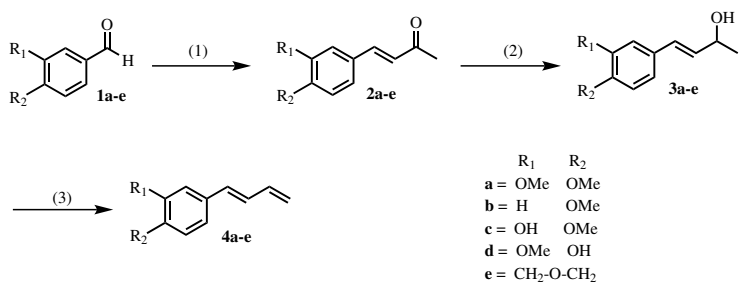

(b)

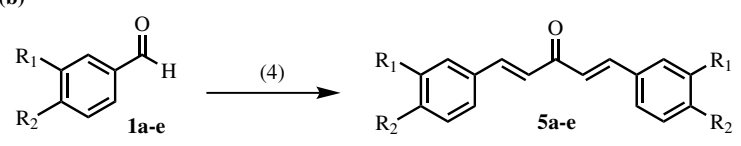

Fig. 1 Synthesis of phenylbutanoid and dienone derivatives. Reagents and conditions: (a) synthesis of phenylbutanoids 4a-e, (1) acetone, $10 \% \mathrm{NaOH}$, rt; (2) $\mathrm{NaBH}_{4}$, methanol, rt; (3) $50 \% \mathrm{H}_{2} \mathrm{SO}_{4}$, dioxane, $40^{\circ} \mathrm{C}$; and (b) synthesis of dienones $5 \mathrm{a}-\mathrm{e}$, (4) acetone ( $0.5 \mathrm{eq}), 10 \% \mathrm{NaOH}$, ethanol.

1a-e and acetone in one step. The Aldol condensation occurred two times in this process. To avoid the formation of excess ketone product, the Aldol condensation of aldehyde to dienone was controlled by using 0.5 equivalent of acetone to react with an equivalent of aldehyde, and compounds $5 \mathrm{a}-\mathrm{e}$ were obtained in $20-99 \%$ yields (Fig. 1). The synthesized compounds were then characterized by FT-IR, ${ }^{1} \mathrm{H}$ NMR, ${ }^{13} \mathrm{C}$-NMR and HRMS.

The ${ }^{1} \mathrm{H}$-NMR coupling constants $(J)$ of the diene side chains were $15-17 \mathrm{~Hz}$, indicating the geometry of $4 \mathrm{a}-\mathrm{e}$ double bond as trans-diene [20]. The ${ }^{1} \mathrm{H}$ NMR of compounds 5a-e showed that the chemical shifts of substitution on carbonyl group of dienone were equivalent due to the symmetrical structures $[18,21]$.

\section{Compounds Characterization}

The synthesis of (E)-4-(substituted phenyl)but-3 -en-2-one (2a-e)

(E)-4-(3,4-dimethoxyphenyl)but-3-en-2-one (2a)

Yellow solid (88\%), $\mathrm{mp}=80.0-81.0^{\circ} \mathrm{C}$. IR (KBr) $\mathrm{cm}^{-1}$ : 2970, 2841, 1701, 1655, 1587, 1455, 1359, 1239, 1016, 969, 875, 801. ${ }^{1} \mathrm{H}-\mathrm{NMR}(400 \mathrm{MHz}$, $\left.\mathrm{CDCl}_{3}\right) \delta: 7.46(\mathrm{~d}, J=16.2 \mathrm{~Hz}, 1 \mathrm{H}), 7.13(\mathrm{dd}$, $J=8.3,2.0 \mathrm{~Hz}, 1 \mathrm{H}), 7.07(\mathrm{~d}, J=1.9 \mathrm{~Hz}, 1 \mathrm{H})$, $6.88(\mathrm{~d}, J=8.3 \mathrm{~Hz}, 1 \mathrm{H}), 6.61(\mathrm{~d}, J=16.2 \mathrm{~Hz}$, 1H), 3.92 (s, 6H), 2.37 (s, 3H). ${ }^{13} \mathrm{C}-\mathrm{NMR}(100 \mathrm{MHz}$, $\mathrm{CDCl}_{3}$ ) $\delta: 198.42,151.41,149.22,143.51,127.33$, 125.27, 123.00, 111.08, 109.61, 55.94, 55.89, 27.34. HRMS (APCI): $\mathrm{m} / \mathrm{z}$ calculated for $\mathrm{C}_{12} \mathrm{H}_{15} \mathrm{O}_{3}$ $\left([\mathrm{M}+\mathrm{H}]^{+}\right):$207.2458, observed value: 207.1005. 


\section{(E)-4-(4-methoxyphenyl)but-3-en-2-one (2b)}

White solid $(81 \%), \mathrm{mp}=73.0-74.0^{\circ} \mathrm{C}$. IR $(\mathrm{KBr})$ $\mathrm{cm}^{-1}: 2919,2843,1588,1457,1359,1235,1163$, 1018, 967, 803. ${ }^{1}$ H-NMR (400 MHz, DMSO-d 6 ) $\delta$ : 7.71-7.62 (m, 2H), 7.57 (d, $J=16.4 \mathrm{~Hz}, 1 \mathrm{H}), 7.01-$ $6.94(\mathrm{~m}, 2 \mathrm{H}), 6.66$ (d, $J=16.3 \mathrm{~Hz}, 1 \mathrm{H}), 3.79$ (s, $3 \mathrm{H}), 2.29$ (s, 3H). ${ }^{13} \mathrm{C}-\mathrm{NMR}$ (100 MHz, DMSO-d 6 ) $\delta: 197.98,161.19,143.15,130.23,126.94,125.06$, 114.48, 55.36, 27.20. HRMS (APCI): $\mathrm{m} / \mathrm{z}$ calculated for $\mathrm{C}_{11} \mathrm{H}_{13} \mathrm{O}_{2}\left([\mathrm{M}+\mathrm{H}]^{+}\right): 177.2198$, observed value: 177.0901 .

\section{(E)-4-(3-hydroxy-4-methoxyphenyl)but-3-en-2- one (2c)}

Pale yellow solid $(94 \%), \mathrm{mp}=79.0-80.0^{\circ} \mathrm{C}$. IR (KBr) $\mathrm{cm}^{-1}: 3717,3137,2926,2845,1631,1582$, 1432, 1361, 1245, 1019, 979, 878, 805. ${ }^{1} \mathrm{H}-\mathrm{NMR}$ (400 MHz, $\mathrm{CDCl}_{3}$ ) $\delta: ~ 7.44-7.40(\mathrm{~d}, J=16.2 \mathrm{~Hz}$, $1 \mathrm{H}), 7.14(\mathrm{~d}, J=2.1 \mathrm{~Hz}, 1 \mathrm{H}), 7.06-7.03$ (dd, $J=$ 8.4, $2.1 \mathrm{~Hz}, 1 \mathrm{H}), 6.86-6.84(\mathrm{~d}, J=8.3 \mathrm{~Hz}, 1 \mathrm{H})$, 6.59-6.55 (d, $J=16.2 \mathrm{~Hz}, 1 \mathrm{H}), 3.91(\mathrm{~d}, J=\mathrm{Hz}$, $3 \mathrm{H}), 2.34(\mathrm{~s}, 3 \mathrm{H}) .{ }^{13} \mathrm{C}-\mathrm{NMR}\left(100 \mathrm{MHz}, \mathrm{CDCl}_{3}\right.$ ) $\delta$ : $198.38,148.71,145.93,143.34,128.01,126.48$, 122.07, 113.14, 112.86, 56.00, 27.48. HRMS (APCI): $\mathrm{m} / \mathrm{z}$ calculated for $\mathrm{C}_{11} \mathrm{H}_{13} \mathrm{O}_{3}\left([\mathrm{M}+\mathrm{H}]^{+}\right)$: 193.2192, observed value: 193.0873.

\section{(E)-4-(4-hydroxy-3-methoxyphenyl)but-3-en-2- one (2d)}

yellow solid $(79 \%), \mathrm{mp}=127.0-128.0^{\circ} \mathrm{C}$. IR $(\mathrm{KBr})$ $\mathrm{cm}^{-1}$ : 3283, 3244, 3033, 2993, 1670, 1631, 1454, 1367, 1260, 1016, 977, 876, 824. ${ }^{1} \mathrm{H}-\mathrm{NMR}$ (400 MHz, $\mathrm{CDCl}_{3}$ ) $\delta: ~ 7.47-7.43$ (d, $J=16.2 \mathrm{~Hz}$, $1 \mathrm{H}$ ), 7.09 (dd, $J=8.2,1.9 \mathrm{~Hz}, 1 \mathrm{H}$ ), 7.06 (d, $J=$ $1.9 \mathrm{~Hz}, 1 \mathrm{H}), 6.94-6.92(\mathrm{~d}, J=8.1 \mathrm{~Hz}, 1 \mathrm{H}), 6.61-$ 6.57 (d, $J=16.2 \mathrm{~Hz}, 1 \mathrm{H}$ ), 5.89 (s, 1H), 3.94 (s, 3H), 2.37 (s, 3H). ${ }^{13} \mathrm{C}-\mathrm{NMR}\left(100 \mathrm{MHz}, \mathrm{CDCl}_{3}\right.$ ) $\delta: 198.37$, $148.22,146.85,143.69,126.93,125.02,123.51$, $114.78,109.26,55.95,27.30$. HRMS (APCI): $\mathrm{m} / \mathrm{z}$ calculated for $\mathrm{C}_{11} \mathrm{H}_{13} \mathrm{O}_{3}\left([\mathrm{M}+\mathrm{H}]^{+}\right)$: 193.2192, observed value: 193.0870 .

\section{(E)-4-(3,4-methylenedioxyphenyl)but-3-en-2-one (2e)}

yellow solid (95\%), $\mathrm{mp}=102.0-104.0^{\circ} \mathrm{C}$. IR $(\mathrm{KBr})$ $\mathrm{cm}^{-1}$ : 2972, 2904, 1702, 1609, 1436, 1236, 1031, 975, 875, 804. ${ }^{1} \mathrm{H}-\mathrm{NMR}\left(400 \mathrm{MHz}, \mathrm{CDCl}_{3}\right.$ ) $\delta$ : 7.45-7.41 (d, $J=16.2 \mathrm{~Hz}, 1 \mathrm{H}), 7.05-7.02(\mathrm{~m}$, $2 \mathrm{H}), 6.83-6.81$ (d, $J=8.0 \mathrm{~Hz}, 1 \mathrm{H}), 6.58-6.54(\mathrm{~d}$, $J=16.2 \mathrm{~Hz}, 1 \mathrm{H}), 6.02(\mathrm{~s}, 2 \mathrm{H}), 2.35(\mathrm{~s}, 3 \mathrm{H}) .{ }^{13} \mathrm{C}-$ NMR (100 MHz, $\mathrm{CDCl}_{3}$ ) $\delta: 198.26,149.85,148.44$, $143.22,128.82,125.29,124.82,108.63,106.52$,
101.62, 27.53. HRMS (APCI): $\mathrm{m} / \mathrm{z}$ calculated for $\mathrm{C}_{11} \mathrm{H}_{11} \mathrm{O}_{3}\left([\mathrm{M}+\mathrm{H}]^{+}\right): 191.2033$, observed value: 191.0711 .

The synthesis of (E)-4-(substituted phenyl)but3-en-2-ol (3a-e)

\section{(E)-4-(3,4-dimethoxyphenyl)but-3-en-2-ol (3a)}

Colourless oil (92\%). IR (KBr) cm $\mathrm{cm}^{-1}: 3316,3061$, 2966, 2839, 1649, 1589, 1454, 1236, 1060, 958, 866, 801. ${ }^{1} \mathrm{H}-\mathrm{NMR}\left(400 \mathrm{MHz}, \mathrm{CDCl}_{3}\right)$ ): $6.94(\mathrm{~d}$, $J=1.9 \mathrm{~Hz}, 1 \mathrm{H}), 6.91(\mathrm{dd}, J=8.2,2.0 \mathrm{~Hz}, 1 \mathrm{H}$ ), $6.81(\mathrm{~d}, J=8.2 \mathrm{~Hz}, 1 \mathrm{H}), 6.50(\mathrm{~d}, J=15.9 \mathrm{~Hz}$, $1 \mathrm{H}), 6.13$ (dd, $J=15.9,6.6 \mathrm{~Hz}, 1 \mathrm{H}), 4.51-4.44$ (m, $1 \mathrm{H}), 3.90$ (s, 3H), 3.88 (s, 3H), 1.37 (d, $J=6.4$ $\mathrm{Hz}, 3 \mathrm{H}) .{ }^{13} \mathrm{C}-\mathrm{NMR}\left(100 \mathrm{MHz}, \mathrm{CDCl}_{3}\right.$ ) $\delta: 149.02$, $148.85,131.61,129.71,129.27,119.67,111.09$, 108.74, 69.04, 55.90, 55.80, 23.47. HRMS (APCI): $\mathrm{m} / \mathrm{z}$ calculated for $\mathrm{C}_{12} \mathrm{H}_{17} \mathrm{O}_{3}\left([\mathrm{M}+\mathrm{H}]^{+}\right): 209.2536$, observed value: 209.1130 .

\section{(E)-4-(4-methoxyphenyl)but-3-en-2-ol (3b)}

Pale yellow solid $(85 \%), \mathrm{mp}=69.0-70.0^{\circ} \mathrm{C}$. IR $(\mathrm{KBr}) \mathrm{cm}^{-1}: 3776,3242,3023,2961,2920,1655$, 1603，1457, 1247, 1063, 963, 810. ${ }^{1}$ H-NMR (400 MHz, $\mathrm{CDCl}_{3}$ ) $\delta: ~ 7.33-7.30(\mathrm{~m}, 2 \mathrm{H}), 6.87-6.84$ (m, 2H), 6.53-6.49 (d, $J=15.9 \mathrm{~Hz}, 1 \mathrm{H}), 6.15-$ 6.10 (dd, $J=15.9,6.6 \mathrm{~Hz}, 1 \mathrm{H}$ ), $4.50-4.44$ (m, $1 \mathrm{H}), 3.81$ (s, 3H), $1.37-1.36$ (d, $J=6.4 \mathrm{~Hz}, 3 \mathrm{H})$. ${ }^{13} \mathrm{C}-\mathrm{NMR}\left(100 \mathrm{MHz}, \mathrm{CDCl}_{3}\right) \delta: 159.26,131.37$, 129.40, 129.03, 127.62, 113.99, 69.13, 55.29, 23.45. HRMS (APCI): $\mathrm{m} / \mathrm{z}$ calculated for $\mathrm{C}_{11} \mathrm{H}_{15} \mathrm{O}_{2}$ $\left([\mathrm{M}+\mathrm{H}]^{+}\right):$179.2357, observed value: 179.0979 .

\section{(E)-4-(3-hydroxy-4-methoxyphenyl)but-3-en-2-ol (3c)}

Pale orange solid $(84 \%), \mathrm{mp}=103.0-104.0^{\circ} \mathrm{C}$. IR $(\mathrm{KBr}) \mathrm{cm}^{-1}: 3463,3131,2961,2921,2852,1678$, 1586, 1444, 1252, 1027, 968, 871, 802. ${ }^{1} \mathrm{H}-\mathrm{NMR}$ (400 MHz, $\mathrm{CDCl}_{3}$ ) $\delta: ~ 7.01-7.00(\mathrm{~d}, J=2.0 \mathrm{~Hz}, 1 \mathrm{H})$, 6.86-6.84 (dd, $J=8.3,2.0 \mathrm{~Hz}, 1 \mathrm{H}), 6.82-6.69$ (m, 2H), 6.48-6.44 (d, $J=15.9 \mathrm{~Hz}, 1 \mathrm{H}), 6.14-6.09$ (dd, $J=15.9,6.5 \mathrm{~Hz}, 1 \mathrm{H}), 5.59(\mathrm{~s}, 1 \mathrm{H}), 4.49-4.43(\mathrm{~m}$, $1 \mathrm{H}), 3.89$ (s, 3H), $1.37-1.35$ (d, $J=6.4 \mathrm{~Hz}, 3 \mathrm{H}$ ). ${ }^{13} \mathrm{C}$-NMR $\left(100 \mathrm{MHz}, \mathrm{CDCl}_{3}\right.$ ) $\delta: 146.34,145.67$, $131.92,129.05,125.48,118.97,111.96,110.54$, 69.03, 55.97, 23.42. HRMS (APCI): $\mathrm{m} / \mathrm{z}$ calculated for $\mathrm{C}_{11} \mathrm{H}_{15} \mathrm{O}_{3}\left([\mathrm{M}+\mathrm{H}]^{+}\right): 195.2351$, observed value: 195.0977. 
(E)-4-(4-hydroxy-3-methoxyphenyl)but-3-en-2-ol (3d)

Yellow oil (81\%). IR (KBr) cm $\mathrm{cm}^{-1}: 3400,2970$, 2931, 1600,1456, 1266, 1219, 1031, 962, 860, 806. ${ }^{1} \mathrm{H}-\mathrm{NMR}\left(400 \mathrm{MHz}, \mathrm{CDCl}_{3}\right.$ ) $\delta: 6.91-6.85$ (m, $3 \mathrm{H}$ ), 6.51-6.46 (dd, $J=15.9,1.0 \mathrm{~Hz}, 1 \mathrm{H}$ ), 6.146.08 (dd, $J=15.8,6.6 \mathrm{~Hz}, 1 \mathrm{H}), 5.65(\mathrm{~s}, 1 \mathrm{H})$, 4.50-4.44 (m, 1H), 3.91 (s, 3H), 1.38-1.36 (d, $J=$ $6.4 \mathrm{~Hz}, 3 \mathrm{H}) .{ }^{13} \mathrm{C}-\mathrm{NMR}\left(100 \mathrm{MHz}, \mathrm{CDCl}_{3}\right) \delta: 146.61$, $145.50,131.25,130.84,125.00,120.29,114.41$, 108.21, 69.06, 55.85, 23.45. HRMS (APCI): $\mathrm{m} / \mathrm{z}$ calculated for $\mathrm{C}_{11} \mathrm{H}_{13} \mathrm{O}_{3}\left([\mathrm{M}+\mathrm{H}]^{+}\right): 193.2129$, observed value: 193.0865 .

\section{(E)-4-(3,4-methylenedioxyphenyl)but-3-en-2-ol (3e)}

Brown oil (85\%). IR (KBr) cm $\mathrm{cm}^{-1}$ : 3348, 2970, 2886, $1650,1608,1490,1242,1034,961,863,799 .{ }^{1} \mathrm{H}-$ $\operatorname{NMR}\left(400 \mathrm{MHz}, \mathrm{CDCl}_{3}\right) \delta: 6.92(\mathrm{~d}, J=1.7 \mathrm{~Hz}, 1 \mathrm{H})$, 6.82-6.79 (dd, $J=8.0,1.6 \mathrm{~Hz}, 1 \mathrm{H}), 6.78-6.71(\mathrm{~m}$, $2 \mathrm{H}$ ), 6.49-6.45 (d, $J=16.2 \mathrm{~Hz}, 1 \mathrm{H}), 6.11-6.06$ (dd, $J=15.8,6.5 \mathrm{~Hz}, 1 \mathrm{H}), 5.95$ (s, 2H), 4.49-4.42 (pd, $J=6.4,1.1 \mathrm{~Hz}, 1 \mathrm{H}), 1.36-1.35$ (d, $J=6.4 \mathrm{~Hz}, 3 \mathrm{H})$. ${ }^{13} \mathrm{C}-\mathrm{NMR}\left(100 \mathrm{MHz}, \mathrm{CDCl}_{3}\right.$ ) $\delta: 148.00,147.23$, $131.79,129.11,121.10,108.26,105.71,101.04$, 68.93, 23.44. HRMS (APCI): $\mathrm{m} / \mathrm{z}$ calculated for $\mathrm{C}_{11} \mathrm{H}_{11} \mathrm{O}_{3}\left([\mathrm{M}+\mathrm{H}]^{+}\right):$191.2032, observed value: 191.0713.

\section{The synthesis of (E)-1-(3,4-substituted phenyl) butadiene (4a-e)}

\section{(E)-1-(3,4-dimethoxyphenyl)butadiene (4a)}

Colourless oil (45\%). IR (KBr) cm $\mathrm{cm}^{-1}: 3079,3001$, 2927, 2837, 1632, 1592, 1454, 1260, 1013, 948, 875, 802. ${ }^{1} \mathrm{H}-\mathrm{NMR}\left(400 \mathrm{MHz}, \mathrm{CDCl}_{3}\right.$ ) $\delta: 6.95$ (dd, $J=11.4,1.9 \mathrm{~Hz}, 2 \mathrm{H}), 6.82(\mathrm{~d}, J=8.1 \mathrm{~Hz}, 1 \mathrm{H})$, 6.67 (dd, $J=15.0,10.9 \mathrm{~Hz}, 1 \mathrm{H}), 6.50$ (dt, $J=18.1$, $6.9 \mathrm{~Hz}, 2 \mathrm{H}), 5.30(\mathrm{~d}, J=16.2 \mathrm{~Hz}, 1 \mathrm{H}), 5.13(\mathrm{~d}$, $J=8.6 \mathrm{~Hz}, 1 \mathrm{H}), 3.91(\mathrm{~s}, 3 \mathrm{H}), 3.89$ (s, 3H). ${ }^{13} \mathrm{C}-$ NMR (100 MHz, $\mathrm{CDCl}_{3}$ ) $\delta: 149.05,148.91,137.23$, $132.62,130.23,127.86,119.83,116.64,111.14$, 108.63, 55.91, 55.80. HRMS (ESI): $\mathrm{m} / \mathrm{z}$ calculated for $\mathrm{C}_{12} \mathrm{H}_{14} \mathrm{NaO}_{2}\left([\mathrm{M}+\mathrm{H}]^{+}\right): 213.2284$, observed value: 213.0889 .

\section{(E)-1-(4-methoxyphenyl)butadiene (4b)}

Pale yellow solid $(30 \%), \mathrm{mp}=48.0-49.0^{\circ} \mathrm{C}$. IR (KBr) $\mathrm{cm}^{-1}: 2921,2846,1674,1602,1454,1243$, 1109, 969, 820. ${ }^{1} \mathrm{H}-\mathrm{NMR}\left(400 \mathrm{MHz}, \mathrm{CDCl}_{3}\right.$ ) б: 7.36-7.33 (m, 2H), 6.88-6.84 (m, 2H), 6.706.64 (dd, $J=15.4,10.5 \mathrm{~Hz}, 1 \mathrm{H}), 6.54-6.47$ (m, $2 \mathrm{H}), 5.30-5.26(\mathrm{~m}, 1 \mathrm{H}), 5.13-5.10(\mathrm{~m}, 1 \mathrm{H}), 3.81$ (s, 3H). ${ }^{13} \mathrm{C}-\mathrm{NMR}\left(100 \mathrm{MHz}, \mathrm{CDCl}_{3}\right.$ ) $\delta: 159.28$, 137.35, 132.38, 129.92, 127.63, 116.43, 114.06, 55.29. HRMS (ESI): $\mathrm{m} / \mathrm{z}$ calculated for $\mathrm{C}_{11} \mathrm{H}_{12} \mathrm{NaO}$ $\left([\mathrm{M}+\mathrm{H}]^{+}\right)$: 183.2024, observed value: 183.0778.

\section{(E)-1-(3-hydroxy-4-methoxyphenyl)butadiene (4c)}

Yellow semi solid (10\%). IR (KBr) $\mathrm{cm}^{-1}$ : 3418, 3078, 3004, 2920, 2847, 1586, 1446, 1269, 1015, 961, 881, 801. ${ }^{1} \mathrm{H}-\mathrm{NMR}\left(400 \mathrm{MHz}, \mathrm{CDCl}_{3}\right.$ ) $\delta: 7.04$ 7.03 (d, $J=2.1 \mathrm{~Hz}, 1 \mathrm{H}$ ), 6.89-6.86 (dd, $J=8.3$, $2.1 \mathrm{~Hz}, 1 \mathrm{H}), 6.80-6.78(\mathrm{~d}, J=8.3 \mathrm{~Hz}, 1 \mathrm{H}), 6.68-$ 6.62 (dd, $J=15.9,10.1 \mathrm{~Hz}, 1 \mathrm{H}), 6.52-6.43(\mathrm{~m}$, $2 \mathrm{H}$ ), 5.31-5.26 (m, 1H), 5.13-5.11 (dd, $J=10.3$, $1.1 \mathrm{~Hz}, 1 \mathrm{H}), 3.89$ (s, 3H). ${ }^{13} \mathrm{C}-\mathrm{NMR}(100 \mathrm{MHz}$, $\mathrm{CDCl}_{3}$ ) $\delta: 146.37,145.67,137.22,132.42,128.15$, 119.13, 116.69, 111.80, 110.58, 55.96. HRMS (ESI): $\mathrm{m} / \mathrm{z}$ calculated for $\mathrm{C}_{11} \mathrm{H}_{13} \mathrm{O}_{2}\left([\mathrm{M}+\mathrm{H}]^{+}\right)$: 177.2198, observed value: 177.0899.

\section{(E)-1-(4-hydroxy-3-methoxyphenyl)butadiene (4d)}

Yellow oil (27\%). IR (KBr) cm $\mathrm{cm}^{-1}: 3420,2923,2851$, 1601, 1456, 1261, 1031, 967, 857, 807. ${ }^{1} \mathrm{H}-\mathrm{NMR}$ $\left(400 \mathrm{MHz}, \mathrm{CDCl}_{3}\right) \delta: 6.94(\mathrm{~s}, 1 \mathrm{H}), 6.92-6.87(\mathrm{~m}$, 2H), 6.70-6.63 (m, 1H), 6.55-6.45 (m, 2H), 5.77 (s, $1 \mathrm{H}), 5.33-5.28$ (dd, $J=17.2,1.3 \mathrm{~Hz}, 1 \mathrm{H}), 5.15-$ 5.12 (dd, $J=9.9,1.5 \mathrm{~Hz}, 1 \mathrm{H}$ ), 3.91 (s, $3 \mathrm{H}) .{ }^{13} \mathrm{C}-$ NMR (100 MHz, $\mathrm{CDCl}_{3}$ ) $\delta: 146.60,145.53,137.22$, 132.74, 129.70, 120.38, 116.38, 114.48, 108.10, 55.77. HRMS (ESI): $\mathrm{m} / \mathrm{z}$ calculated for $\mathrm{C}_{11} \mathrm{H}_{12} \mathrm{NaO}_{2}$ $\left([\mathrm{M}+\mathrm{H}]^{+}\right): 199.2018$, observed value: 199.0713.

\section{(E)-1-(3,4-methylenedioxyphenyl)butadiene (4e)}

White semi solid (39). IR (KBr) $\mathrm{cm}^{-1}: 2916,1671$, 1607, 1489, 1239, 1033, 967, 863, 802. ${ }^{1} \mathrm{H}-\mathrm{NMR}$ $\left(400 \mathrm{MHz}, \mathrm{CDCl}_{3}\right) \delta: 6.97-6.96(\mathrm{~d}, J=1.7 \mathrm{~Hz}, 1 \mathrm{H})$, 6.86-6.83 (dd, $J=8.0,1.6 \mathrm{~Hz}, 1 \mathrm{H}), 6.78-6.76$ (d, $J=8.0 \mathrm{~Hz}, 1 \mathrm{H}$ ), 6.67-6.61 (m, 1H), 6.53-6.44 (m, $2 \mathrm{H}$ ), 5.95 (s, 2H), 5.33-5.28 (dd, $J=17.4,1.4 \mathrm{~Hz}$, $1 \mathrm{H}), 5.16-5.13(\mathrm{dd}, J=9.9,1.5 \mathrm{~Hz}, 1 \mathrm{H}) .{ }^{13} \mathrm{C}-$ NMR (100 MHz, $\left.\mathrm{CDCl}_{3}\right) \delta: 148.03,147.26,137.11$, $132.47,131.60,127.97,121.32,116.81,108.30$, 105.46, 101.03. HRMS (ESI): $\mathrm{m} / \mathrm{z}$ calculated for $\mathrm{C}_{11} \mathrm{H}_{11} \mathrm{O}_{2}\left([\mathrm{M}+\mathrm{H}]^{+}\right): 175.2039$, observed value: 175.0655 . 
The synthesis of $(1 E, 4 E)-1,5$-bis(substituted phenyl)penta-1,4-dien-3-one (5a-e)

(1E,4E)-1,5-bis(3,4-dimethoxyphenyl)penta-1,4dien-3-one (5a)

Yellow solid (48\%), $\mathrm{mp}=198.0-199.0^{\circ} \mathrm{C}$. IR $(\mathrm{KBr})$ $\mathrm{cm}^{-1}$ : 2920, 2851, 1703, 1592, 1455 1248, 1020, 965, 856, 807. ${ }^{1} \mathrm{H}-\mathrm{NMR}\left(400 \mathrm{MHz}, \mathrm{CDCl}_{3}\right.$ ) $\delta: 7.70$ (d, $J=15.8 \mathrm{~Hz}, 1 \mathrm{H}), 7.21(\mathrm{dd}, J=8.3,1.8 \mathrm{~Hz}, 1 \mathrm{H})$, 7.15 (d, $J=1.8 \mathrm{~Hz}, 1 \mathrm{H}), 6.96$ (d, $J=15.8 \mathrm{~Hz}, 1 \mathrm{H})$, 6.90 (d, $J=8.3 \mathrm{~Hz}, 1 \mathrm{H}), 3.95$ (s, 3H), 3.93 (s, 3H). ${ }^{13} \mathrm{C}$-NMR $\left(100 \mathrm{MHz}, \mathrm{CDCl}_{3}\right.$ ) $\delta: 188.70,151.36$, $149.27,143.05,127.87,123.64,123.10,111.12$, 109.91, 56.00, 55.95. HRMS (ESI): $\mathrm{m} / \mathrm{z}$ calculated for $\mathrm{C}_{21} \mathrm{H}_{23} \mathrm{O}_{5}\left([\mathrm{M}+\mathrm{H}]^{+}\right): 355.4044$, observed value: 355.1528 .

(1E,4E)-1,5-bis(4-methoxyphenyl)penta-1,4dien-3-one ( $5 b$ )

Yellow solid (99\%), mp $=124.0-125.0^{\circ} \mathrm{C}$. IR $(\mathrm{KBr})$ $\mathrm{cm}^{-1}:$ 3020, 2954, 2837, 1597, 1455, 1249, 1027, 977, 825. ${ }^{1} \mathrm{H}-\mathrm{NMR}$ (400 MHz, DMSO-d 6 ) $\delta: 7.75$ 7.70 (dd, $J=12.3,9.2 \mathrm{~Hz}, 6 \mathrm{H}$ ), $7.21-7.17$ (d, $J=$ $16.0 \mathrm{~Hz}, 2 \mathrm{H}), 7.03-7.01$ (d, $J=8.8 \mathrm{~Hz}, 4 \mathrm{H}), 3.81$ (s, 6H). ${ }^{13} \mathrm{C}-\mathrm{NMR}(100 \mathrm{MHz}$, DMSO-d 6 ) $\delta: 188.22$, 161.22, 142.22, 130.34, 127.39, 123.58, 114.50, 55.39. HRMS (ESI): $\mathrm{m} / \mathrm{z}$ calculated for $\mathrm{C}_{19} \mathrm{H}_{18} \mathrm{NaO}_{3}$ $\left([\mathrm{M}+\mathrm{H}]^{+}\right): 317.3344$, observed value: 317.1125 .

\section{(1E,4E)-1,5-bis(3-hydroxy-4-methoxyphenyl)} penta-1,4-dien-3-one (5c)

Yellow solid (20\%), $\mathrm{mp}=193.0-194.0^{\circ} \mathrm{C}$. IR $(\mathrm{KBr})$ $\mathrm{cm}^{-1}: 3412,2948,2838,1651,1594,1435,1272$, 1024, 977, 872, 802. ${ }^{1} \mathrm{H}-\mathrm{NMR}\left(400 \mathrm{MHz}, \mathrm{CDCl}_{3}\right.$ ) $\delta$ : $7.66-7.62$ (d, $J=15.8 \mathrm{~Hz}, 2 \mathrm{H}), 7.24$ (d, $J=1.8 \mathrm{~Hz}$, $2 \mathrm{H}$ ), 7.13-7.10 (dd, $J=8.4,1.8 \mathrm{~Hz}, 2 \mathrm{H}), 6.95$ 6.86 (dd, $J=21.3,12.1 \mathrm{~Hz}, 4 \mathrm{H}), 3.94(\mathrm{~s}, 6 \mathrm{H}) .{ }^{13} \mathrm{C}-$ NMR (100 MHz, $\mathrm{CDCl}_{3}$ ) $\delta: ~ 198.51,151.07,145.8$ $9,142.85,128.57,124.00,122.47,113.02,110.58$, 56.02. HRMS (ESI): $\mathrm{m} / \mathrm{z}$ calculated for $\mathrm{C}_{19} \mathrm{H}_{18} \mathrm{NaO}_{5}$ $\left([\mathrm{M}+\mathrm{H}]^{+}\right): 349.3332$, observed value: 349.1055 .

\section{(1E,4E)-1,5-bis(4-hydroxy-3-methoxyphenyl) penta-1,4-dien-3-one (5d)}

Yellow semi solid (45\%), IR (KBr) $\mathrm{cm}^{-1}$ : 3372 , 2924, 2853, 1711, 1630, 1586, 1457, 1262, 1027, 981, 854, 812. ${ }^{1} \mathrm{H}-\mathrm{NMR}\left(400 \mathrm{MHz}, \mathrm{CDCl}_{3}\right.$ ) $\delta: 7.67$ (d, $J=15.7 \mathrm{~Hz}, 1 \mathrm{H}), 7.19$ (dd, $J=8.1,1.8 \mathrm{~Hz}$, $1 \mathrm{H}), 7.12(\mathrm{~d}, J=1.6 \mathrm{~Hz}, 1 \mathrm{H}), 6.96(\mathrm{~d}, J=2.3 \mathrm{~Hz}$, $1 \mathrm{H}), 6.93(\mathrm{~d}, J=9.9 \mathrm{~Hz}, 1 \mathrm{H}), 3.96(\mathrm{~s}, 3 \mathrm{H}) .{ }^{13} \mathrm{C}-$ NMR (100 MHz, $\mathrm{CDCl}_{3}$ ) $\delta: 189.06,148.26,146.86$, $143.48,127.37,123.37,123.18,114.88,109.88$,
Table 1 Percentage of cell viability and $\mathrm{IC}_{50}$ of synthesized compounds on RAW264.7 cells.

\begin{tabular}{lcc}
\hline Compound & \% Cell viability at $15.63 \mu \mathrm{M}$ & $\mathrm{IC}_{50}(\mu \mathrm{M})$ \\
\hline Control & $100.00 \pm 0.01$ & \\
MMC & $32.27 \pm 0.12$ & \\
4a & $91.48 \pm 0.02$ & $103.28 \pm 0.15$ \\
4b & $87.42 \pm 0.03$ & $294.79 \pm 1.12$ \\
4c & $81.85 \pm 0.02$ & $184.08 \pm 0.09$ \\
4d & $85.70 \pm 0.14$ & $113.59 \pm 0.64$ \\
4e & $86.41 \pm 0.06$ & $243.94 \pm 0.09$ \\
5a & $89.44 \pm 0.17$ & $178.85 \pm 0.27$ \\
5b & $89.44 \pm 0.23$ & $213.18 \pm 0.74$ \\
5c & $87.98 \pm 0.54$ & $280.51 \pm 0.14$ \\
5d & $88.43 \pm 0.30$ & $266.98 \pm 0.32$ \\
5e & $93.50 \pm 0.51$ & $273.06 \pm 0.09$ \\
diclofenac & $83.87 \pm 0.08$ & $112.67 \pm 0.62$ \\
Plai oil ${ }^{\mathrm{a}}$ & $89.45 \pm 0.25$ & $285.09 \pm 0.14$ \\
\hline
\end{tabular}

Each value is mean $\pm \mathrm{SE}(n=3)$. $\mathrm{IC}_{50}$ is the concentration that inhibited cell growth by $50 \%$. MMC is the highly cytotoxic compound Mitomycin C.

${ }^{a}$ is the essential oil from $Z$. cassumunar Roxb. containing 30\% (E)-4-(3,4-dimethoxyphenyl)butadiene at.

55.94. HRMS (ESI): $\mathrm{m} / \mathrm{z}$ calculated for $\mathrm{C}_{19} \mathrm{H}_{19} \mathrm{O}_{5}$ $\left([\mathrm{M}+\mathrm{H}]^{+}\right)$: 327.3512, observed value: 327.1201.

\section{(1E,4E)-1,5-bis(3,4-methylenedioxyphenyl) penta-1,4-dien-3-one (5e)}

Yellow solid $(79 \%), \mathrm{mp}=173.0-174.0^{\circ} \mathrm{C}$. IR $(\mathrm{KBr})$ $\mathrm{cm}^{-1}$ : 3066, 3004, 2902, 1706, 1640, 1587, 1443, 1253, 1033, 927, 876, 806. ${ }^{1} \mathrm{H}-\mathrm{NMR}$ (400 MHz, $\mathrm{CDCl}_{3}$ ) $\delta: 7.66-7.62$ (d, $\left.J=15.8 \mathrm{~Hz}, 2 \mathrm{H}\right), 7.13-$ 7.12 (d, $J=1.7 \mathrm{~Hz}, 2 \mathrm{H}$ ), 7.10-7.08 (dd, $J=8.1$, $1.6 \mathrm{~Hz}, 2 \mathrm{H}$ ), $6.90-6.83$ (dd, $J=19.0,11.9 \mathrm{~Hz}$, 4H), $6.02(\mathrm{~s}, 4 \mathrm{H}) .{ }^{13} \mathrm{C}-\mathrm{NMR}\left(100 \mathrm{MHz}, \mathrm{CDCl}_{3}\right) \delta$ : 188.56, 149.80, 148.39, 142.83, 129.31, 125.04, 123.75, 108.65, 106.58, 101.59. HRMS (ESI): $\mathrm{m} / \mathrm{z}$ calculated for $\mathrm{C}_{19} \mathrm{H}_{14} \mathrm{NaO}_{5}\left([\mathrm{M}+\mathrm{H}]^{+}\right): 345.3015$, observed value: 345.0711 .

\section{Cytotoxic and anti-inflammatory activities of phenylbutanoid and dienone derivatives}

\section{Cytotoxic activity}

RAW264.7 cells were treated with various concentrations of the samples, ranging from 15.63 to $500 \mu \mathrm{M}$, and evaluated by WST-1 assay using the MMC as a positive control, all compounds exhibited decreased \% cell viability in a dosedependent manner (Table 1 ). The $\mathrm{IC}_{50}$ values were calculated and used as a parameter of cytotoxicity. The results show that $4 \mathrm{~b}$ has the highest $\mathrm{IC}_{50}$ $(294.79 \pm 1.12 \mu \mathrm{M})$, while the lowest is found in $4 \mathrm{a}$ 
Table 2 Nitric oxide levels and percentage of nitric oxide inhibition following treatment with synthesized compounds at $15.63 \mu \mathrm{M}$.

\begin{tabular}{lcc}
\hline Treatment & Nitric oxide $(\mu \mathrm{m})$ & \% Nitric oxide inhibition \\
\hline Control & $11.52 \pm 0.06$ & $\mathrm{NT}$ \\
LPS & $205.24 \pm 0.20$ & $\mathrm{NT}$ \\
4a & $155.51 \pm 0.41$ & $24.23 \pm 0.22$ \\
4b & $52.79 \pm 0.74$ & $74.28 \pm 0.14$ \\
4c & $205.15 \pm 0.06$ & $\mathrm{NE}$ \\
4d & $196.35 \pm 0.10$ & $4.33 \pm 0.35$ \\
4e & $47.64 \pm 0.20$ & $76.79 \pm 0.31$ \\
5a & $32.41 \pm 0.41$ & $84.21 \pm 0.40$ \\
5b & $77.77 \pm 0.18$ & $62.11 \pm 0.33$ \\
5c & $129.31 \pm 0.44$ & $37.00 \pm 0.50$ \\
5d & $139.73 \pm 0.32$ & $31.921 \pm 0.21$ \\
5e & $33.02 \pm 1.66$ & $83.91 \pm 1.09$ \\
Plai oil ${ }^{\mathrm{a}}$ & $36.06 \pm 0.16$ & $82.43 \pm 0.26$ \\
Diclofenac $^{\mathrm{c}}$ & $175.54 \pm 0.29$ & $14.47 \pm 0.59$ \\
\hline
\end{tabular}

Each value is presented as mean \pm SE $(n=3)$; $(p<$ $0.05)$; NT is not tested; NE is not effective.

${ }^{a}$ the essential oil from $Z$. cassumunar Roxb. containing 30\% (E)-4-(3,4-dimethoxyphenyl)butadiene.

$(103.28 \pm 0.15 \mu \mathrm{M})$. Moreover, all samples at a concentration of $15.63 \mu \mathrm{M}$ were potentially non-toxic, as indicated by cell viability of more than $70 \%$ [22]. Therefore, the $15.63 \mu \mathrm{M}$ concentration of samples that exhibited $70 \%$ cell viability against RAW264.7 cell was chosen for the anti-inflammation assay.

\section{Anti-inflammatory activity}

When RAW264.7 cells were pre-treated with samples at a concentration of $15.63 \mu \mathrm{M}$ for $24 \mathrm{~h}$ and then treated with $10 \mu \mathrm{g} / \mathrm{ml}$ LPS, the macrophage cells exhibited high concentration levels of NO (up to $205.24 \pm 0.20 \mu \mathrm{M}$ ) which is due to the proinflammatory LPS.

Compounds 4b, 4e, 5a, 5b, and 5e showed a high percentage of NO inhibition $(62.11 \pm 0.33$ $84.21 \pm 0.40 \%$ ) on LPS-stimulated RAW264.7 macrophage cells. Unfortunately, no anti-inflammatory capacity was found in 4c (Table 2).

The percentage of NO inhibition for compounds $5 \mathrm{a}$ and $5 \mathrm{e}$ are higher than the other compounds, revealing that the methoxy or 1,3-dioxol substituted (E)-1-(3,4-disubstituted phenyl)butadienes and $(1 E, 4 E)$-1,5-bis(3,4-disubstituted phenyl)penta1,4-dien-3-ones tend to show a higher potential for the inhibition of NO production than compounds with one substituent on the aromatic ring $[23,24]$. On the contrary, the compounds substituted by a hydroxy group at any position showed low anti-inflammatory activity. Whereas the identical substituted on benzene ring of 1,4-pentadienones and phenylbutadienes, we found that the 1,4pentadienones showed higher potential than the phenylbutadienes [25].

According to the percentage of inhibition of synthesized compounds, the results showed that the 5a had a higher anti-inflammatory activity than the essential oil from Z. cassumunar Roxb, containing 30\% of DMPBD (4a) and the diclofenac drug [6] with high percentage of cell viability (Tables 1 and 2). The cytotoxic and anti-inflammatory activities of compound 5 a suggest its potential use as an antiinflammatory agent.

\section{CONCLUSION}

Phenylbutanoid (4a-e) and dienone (5a-e) derivatives were synthesized from general reaction, and compounds were obtained in moderate to high yields. Cytotoxic and anti-inflammatory activities of all synthesized compounds were studied under LPS stimulation. Interestingly, compounds $4 \mathrm{~b}, 4 \mathrm{e}, 5 \mathrm{a}$, $5 \mathrm{~b}$, and $5 \mathrm{e}$ showed high anti-inflammatory activity at low concentrations without affecting cell viability of RAW264.7 macrophage cells, and their activity was higher than the diclofenac drug.

Acknowledgements: This work was supported by the Development and Promotion of Science and Technology Talents Project (DPST). We thank all researchers and laboratory members from the Department of Chemistry and the Department of Zoology, Faculty of Science, Kasetsart University for their equipment, knowledge, and technical support.

\section{REFERENCES}

1. Okyar A, Özsoy Y, Güngör S (2012) Novel formulation approaches for dermal and transdermal delivery of non-steroidal anti-inflammatory drugs. In: Lemmey A (ed) Rheumatoid Arthritis - Treatment, InTech, Turkey, pp 25-48.

2. Ong CKS, Lirk P, Tan CH, Seymour RA (2007) An evidence-based update on nonsteroidal antiInflammatory drugs. $J$ Clin Med Res 5, 19-34.

3. Wongrakpanich S, Wongrakpanich A, Melhado K, Rangaswami J (2018) A comprehensive review of non-steroidal anti-inflammatory drug use in the elderly. Aging Dis 9, 143-150.

4. Singh CB, Manglembi N, Swapana N, Chanu SB (2015) Ethnobotany, phyto chemistry and pharmacology of Zingiber cassumunar Roxb. (Zingiberaceae). J Phar macogn Phytochem 4, 1-6.

5. Sukatta U, Rugthaworn P, Punjee P, Chidchenchey S, Keeratinijakal V (2009) Chemical composition and 
physical properties of oil from Plai (Zingiber cassumunar Roxb.) obtained by hydro distillation and hexane extraction. Kasetsart J (Nat Sci) 43, 212-217.

6. Jeenapongsa R, Yoovathaworn K, Sriwatanakul KM, Pongprayoon U, Sriwatanakul K (2003) Anti-inflammatory activity of (E)-1-(3,4-dimethoxyphenyl)butadiene from Zingiber cassumunar Roxb. $J$ Ethnopharmacol 87, 143-148.

7. Ohori H, Yamakoshi H, Tomizawa M, Shibuya M, Kakudo Y, Takahashi A, Takahashi S, Kato S, et al (2006) Synthesis and biological analysis of new curcumin analogues bearing an enhanced potential for the medicinal treatment of cancer. Mol Cancer Ther 5, 2563-2571.

8. Agbaje OC, Fadeyi OO, Okoro CO (2011) Lewis acid mediated diastereoselective synthesis of fused fluorinated spiroketal as potential biologically active compounds. Tetrahedron Lett 52, 5297-5300.

9. Anto JR, Sukumaran K, Kuttan G, Rao MNA, Subbaraju V, Kuttan R (1995) Anticancer and antioxidant activity of synthetic chalcones and related compounds. Cancer Lett 97, 33-37.

10. Flannagan S, Harrison E, Yip M, Jaqaman K, Grinstein J (2007) Macrophages are crucial frontline cells in the body's defense against infection. $J$ Leukoc Biol 82, 417-428.

11. Ariel A, Maridonneau-Parini I, Rovere-Querini $\mathrm{P}$, Levine J, Mühl H (2012) Macrophages in inflammation and its resolution. Front Immunol 324, 1-12.

12. Shaikh Z (2011) Cytokine \& their physiologic and pharmacologic functions in inflammation: a review. Inter J Pharma Life Sci 2, 1247-1263.

13. Soromou W, Zhang R, Li R, Chen N, Guo W, Huo M, Guan S, Lu J, et al (2012) Regulation of inflammatory cytokine in lipopolysachharidestimulated RAW264.7 murine macrophage by 7-Omethyl-naringenin. Molecules 17, 3574-3585.

14. Moorcroft MJ, Davis J, Compton RG (2001) Detection and determination of nitrate and nitrite: a review. Talanta 54, 785-803.

15. Jitoe A, Masuda T, Nakatani N (1993) Phenylbutenoid dimers from the rhizome of Zingiber cassumunar. Phytochemistry 32, 357-363.

16. Tangyuenyongwatana P, Gritsanapan W (2008) A study on artifacts formation in the Thai traditional medicine. Planta Med 74, 761-764.

17. Yogosawa S, Yamada Y, Yasuda S, Sun Q, Takizawa K, Sakai T (2012) Dehydrozingerone, a structural analogue of curcumin, induces cell-cycle arrest at the G2/M phase and accumulates intracellular ROS in HT-29 human colon cancer cells. J Nat Prod 75, 2088-2093.

18. Deck LM, Hunsaker LA, Vander Jagt TA, Whalen LJ, Royer RE, Vander Jagt DL (2017) Activation of anti-oxidant Nrf2 signaling by enone analogues of curcumin. Eur J Med Chem 143, 854-865.

19. Ishiyama M, Shiga M, Sasamoto K, Mizoguchi M, He P (1993) A new sulfonated tetrazolium salt that produces a highly water-soluble formazan dye. Chem Pharm Bull 41, 1118-1122.

20. Tanvi VW, Silvia B, Pravin SK, Abdul HC, Claudiu TS, Mrunmayee PT (2018) Evaluation of sulphonamide derivatives acting as inhibitors of human carbonic anhydrase isoforms I, II and Mycobacterium tuberculosis $\beta$-class enzyme Rv3273. J Enzyme Inhib Med Chem 33, 962-971.

21. Cheng C, Ning X, Luo Y, Tian C, Wang X, Guo Y, Liu J, Zhang Z (2016) Synthesis and neuroprotective evaluation of (E)-3,4-dihydroxystyryl p-substitutedphenethyl ketone derivatives against inflammatory and oxidative injury. Med Chem Res 25, 1678-1685.

22. Dechayont B, Phuaklee P, Chunthorng-Orn J, Poomirat S, Prajuabjinda O, Vilaichone R, Itharat A (2019) Anti-Helicobacter pylori, anti-inflammatory and antioxidant evaluation of crude extracts from Amomum krervanh fruits. ScienceAsia 45, 109-115.

23. Liang G, Li X, Chen L, Yang S, Wu X, Studer E, Gurley E, Hylemon PB, et al (2008) Synthesis and antiinflammatory activities of mono-carbonyl analogues of curcumin. Bioorg Med Chem Lett 18, 1525-1529.

24. Lee KH, Ab Aziz FH, Syahida A, Abas F, Shaari K, Israf DA, Lajis NH (2009) Synthesis and biological evaluation of curcumin-like diarylpentanoid analogues for anti-inflammatory, antioxidant and anti-tyrosinase activities. Eur J Med Chem 44, 3195-3200.

25. Kaewchoothong A, Tewtrakul S, Panichayupakaranant P (2012) Inhibitory effect of phenylbutanoid-rich Zingiber cassumunar extracts on nitric oxide production by murine macrophage-like RAW264.7 cells. Phytother Res 26, 1789-1792. 